\title{
Bipolar disorder: an update
}

\author{
Kim Outhoff \\ Senior Lecturer, Department of Pharmacology, University of Pretoria
}

*Corresponding author, email: kim.outhoff@up.ac.za

\begin{abstract}
Bipolar disorder, characterised by alternating discrete episodes of (hypo)mania and depression, provides unique diagnostic and treatment challenges. Updated diagnostic (DSM-5) and current pharmacological treatment recommendations are briefly reviewed here.
\end{abstract}

Keywords: bipolar disorder; diagnosis; evidence-based pharmacotherapy

\section{Introduction}

Bipolar disorder (BD) is a relatively uncommon ( 1\%-4.5\% lifetime incidence), recurrent mood disorder, with strong genetic undertones, characterised by significant mood swings between depressive and (hypo)manic episodes.1,2 (Figure 1.) Bipolar cycling may be rapid (four mood cycles a year), and periods of euthymia may be elusive. ${ }^{3}$ Although many with BD return to fully functional levels between episodes, up to $30 \%$ show severe impairment in occupational function. ${ }^{4}$ Depression is usually the predominant mood abnormality; the lifetime suicide risk is estimated at 15 times higher than in the general population and BD may account for a quarter of all completed suicides., ${ }^{2,5}$ Co-morbid anxiety and substance misuse are common and may contribute to mood instability. ${ }^{2}$
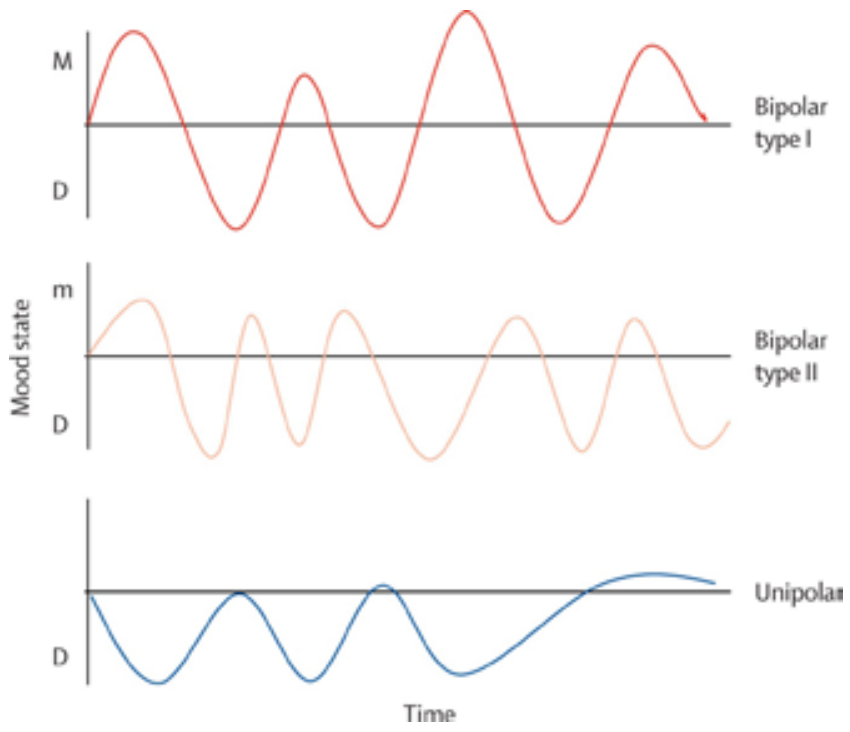

Figure 1. Mood changes over time in bipolar disorder type l, bipolar disorder type II, and recurrent unipolar depressive disorder $\mathrm{M}=$ mania; $\mathrm{m}=$ hypomania; $\mathrm{D}=$ depression. $^{6}$
A manic episode is a distinct period during which there is an abnormally, persistently elevated, expansive, or irritable mood and persistently increased activity or energy that is present for most of the day, nearly every day, for a period of at least one week (or any duration if hospitalisation is necessary), accompanied by at least three additional symptoms of mania, including inflated self-esteem/grandiosity, significantly decreased need for sleep, racing speech, flight of ideas/ideas that move quickly from one subject to the next, easily distracted, increased goal-directed activity and excessive involvement in activities that have a high potential for painful consequences. ${ }^{7}$ Once a patient has experienced a full manic episode, irrespective of the presence or severity of depressive episodes, the illness is classified as BD I. (Figure 1.) By definition, the symptoms of BD I cause clinically significant distress or impairment in important areas of functioning. Psychotic features, usually mood-congruent delusions which occur in approximately $50 \%$ of patients, also demand a diagnosis of BD I. ${ }^{2}$ Substance use or abuse and other medications (adrenergic agents, corticosteroids, conventional antidepressants), and symptoms caused by general medical conditions need to be excluded before diagnosing any phase of $\mathrm{BD}$, including manic episodes. ${ }^{7}$

In hypomania, the duration of symptoms, including elevated mood, is shorter (four consecutive days) than in mania, and the persistently increased activity is not necessarily goal directed. ${ }^{8}$ The episode is not sufficiently severe to cause marked impairment in social or occupational functioning or to necessitate hospitalisation. ${ }^{7}$ (Figure 1.)

A major depressive episode is characterised by a two week period of depressed mood and/or loss of interest or pleasure in almost all activities for most of the day, nearly every day, accompanied by significant weight gain or loss, insomnia or hypersomnia, psychomotor agitation or retardation, loss of energy and 
constant lethargy, sense of guilt or low self-esteem, difficulty concentrating, or recurrent thoughts of suicide or death, causing significant distress or impaired functioning. ${ }^{7}$ When a major depressive episode is associated with hypomanic episodes (in the absence of manic episodes), the illness is classified as BD II. (Figure 1.)

Major depressive episodes, particularly of BD II, may easily be misdiagnosed as unipolar depression, as detecting the subtleties of hypomania may prove difficult. Recently it was reported that approximately $20 \%$ of unipolar depressed patients in the antidepressant clinical trial setting convert to a diagnosis of $\mathrm{BD}$, and that the risk of this diagnostic conversion is positively associated with the number of previous depressive episodes. ${ }^{9}$ Other potential distinguishing clues flagging the possibility of depression of BD include an abrupt and earlier age of onset, hypersomnia, psychotic symptoms, shorter episodes of depression, comorbid substance abuse and strong family history. ${ }^{8}$ Conversely, unipolar depression is more commonly associated with somatic symptoms, anxiety and loss of appetite/ weight than bipolar depressive episodes. ${ }^{9}$ Treatment of bipolar depression with conventional antidepressant agents is often futile (hence perhaps the high relapse rate), and furthermore, may trigger manic episodes that last beyond the pharmacokinetic and biological half lives of these drugs. The importance of a correct diagnosis, and therefore appropriate treatment, is critical, and often falls to a psychiatrist rather than a family practitioner, particularly when there is a risk of suicide, diagnostic uncertainty or severe disability. ${ }^{8}$ However, in a resourcelimited country, this may not always prove practical.

\section{Aetiology/pathophysiology}

The proposed aetiology of mood disorders is complex and involves interplays between genetic predisposition, psychological vulnerability and life stressors, resulting in reinforcing loops of molecular, cellular and neural dysfunction, inner distress and ultimately changed behaviours. ${ }^{10,11}$ (Figure 2.)

Predisposing, precipitating and perpetuating factors as well as poor protective factors contribute significantly to the presenting episode, while strengthening resilience and treatment offers respite. ${ }^{12}$ Identifying these factors during case formulation reinforces the foundation of a successful therapeutic relationship
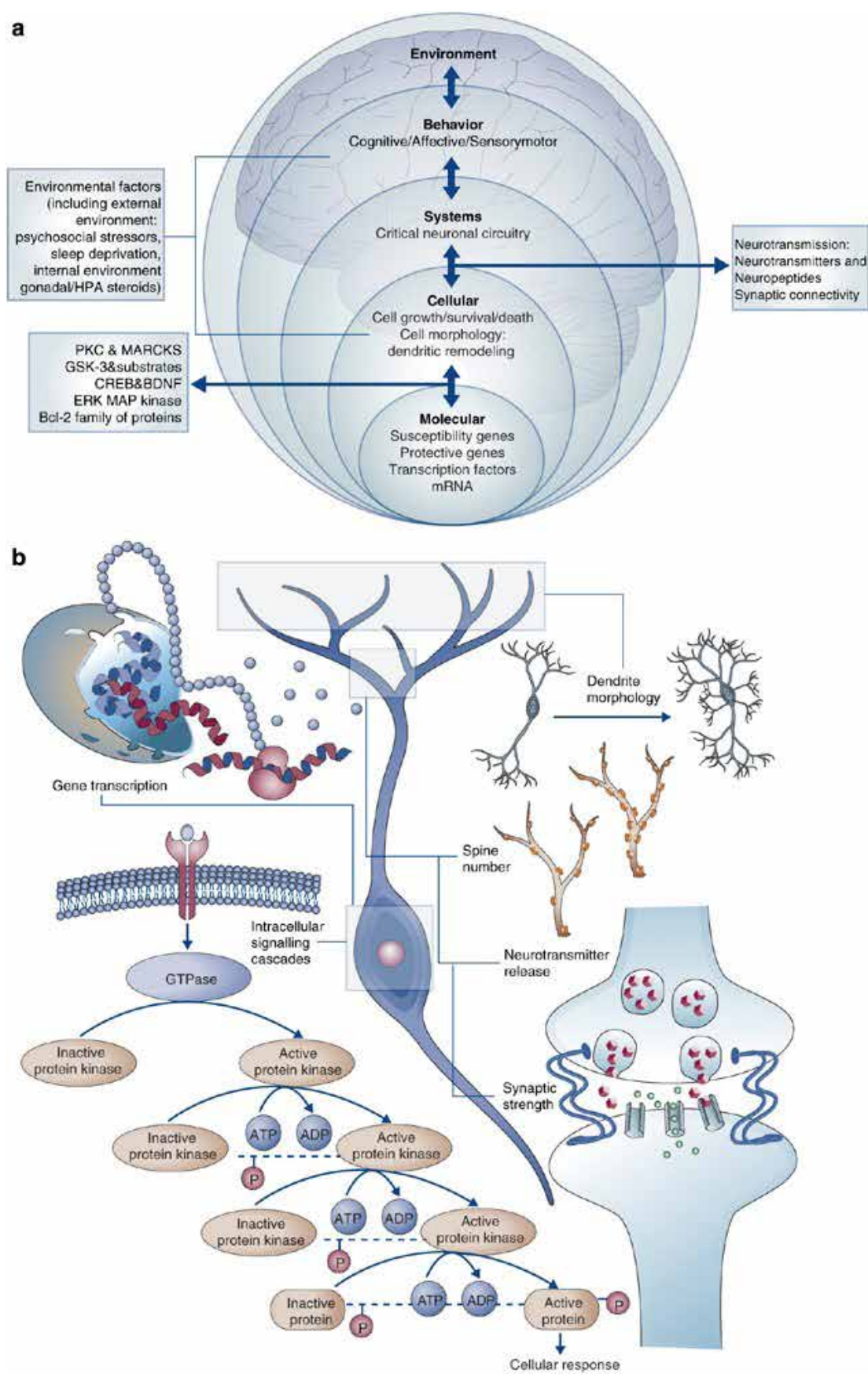

Figure 2. (a) A true understanding of the pathophysiology of BPD must encompass different systems on different physiological levels at which the disease manifests: molecular, cellular, and behavioural. (b) Biological mechanisms underlying neuroplasticity. The remarkable plasticity of neuronal circuits is achieved through different biological means including alterations in gene transcription and intracellular signalling cascades. These changes modify diverse neuronal properties such as neurotransmitter release, synaptic function and even morphological characteristics of neurons. ${ }^{10}$

that is built on understanding individuals in the context of their unique circumstances, and facilitates biopsychosocial treatment interventions at multiple levels. ${ }^{13}$ For instance, resilience, or the ability to adapt to and recover from stress, is a mitigating factor for all three phases of $\mathrm{BD}^{14}$ and can be strengthened biologically (using lithium as a neuroprotective agent), psychologically (teaching cognitive reappraisal skills; online mindfulness-based therapy), socially (improving social support), and through lifestyle change (improving exercise habits). ${ }^{15,8,16}$ That said, 
pharmacotherapy forms the cornerstone of treatment for BD and first-line recommendations are outlined briefly here. ${ }^{17}$

\section{Pharmacological treatment}

Evidence-based treatment guidelines differ slightly between countries depending on usage patterns and availability of licensed drugs. 8,17-20 Lithium, the antiepileptic drugs (valproate and lamotrigine) and the second generation antipsychotics (SGAs) feature prominently. $8,18,19$ Although their mechanisms of action are diverse, these agents contribute to maintaining neuronal integrity and stability. For instance, lithium, valproate and lamotrigine are thought to stabilise neural conduction by modulating dysfunctional voltage gated sodium and calcium channels. ${ }^{1,21}$ Intracellular actions of lithium and valproate include stimulating cell survival pathways and increasing levels of neurotrophic factors to improve cellular resiliency and to provide neuroprotection. ${ }^{22-24}$ Valproate has also been shown to enhance neuro-inhibitory GABA effects ${ }^{25}$ while possibly attenuating neuro-excitatory glutamate's effects by up-regulating calcium chaperone protein, GRP $78 .{ }^{26}$ Valproate's effects on DNA histone acetylation may possibly regulate epigenetic phenomena as well. ${ }^{27}$

The SGAs mechanism of antimanic action is related to antagonising the arousing effects of both serotonin (5HT) and dopamine, prominent neurotransmitter pathways implicated in the pathophysiology of mania.Their mechanism ofantidepressant action is speculative. ${ }^{26}$ Olanzapine and quetiapine, which are both licensed for bipolar depression, antagonise serotonin $5 \mathrm{HT}_{2 \mathrm{~A}}$ receptors while stimulating $5 \mathrm{HT}_{1 \mathrm{~A}}$ receptors and this is thought to contribute to their antidepressant effects. In addition, prefrontal cortical dopamine levels are indirectly elevated by this $5 \mathrm{HT}_{1 \mathrm{~A}}$ partial agonistic mechanism. Rapid dissociation of quetiapine from the dopamine $D_{2}$ receptors as well as altered expression of glutamate receptor subunits may also partially account for its antidepressant efficacy in BD. ${ }^{26}$

The choice and dose of recommended agent/s are based on patient preference, previous response, risk/benefit assessments, clinical utility, concomitant medication and potential drug interactions, safety monitoring of medications including therapeutic drug monitoring, drug adherence, burden of disease (mania vs depression) and phase of illness. ${ }^{28}$

\section{Long-term maintenance}

Maintenance treatment in $\mathrm{BD}$ serves to preserve euthymia and to limit the inexorable increasing frequency and severity of future (hypo)manic or depressive episodes. Lithium, the doyenne and gold-standard treatment for prophylaxis in BD, is particularly effective for maintaining remission (preventing both manic and depressive episodes), conferring anti-suicidal and neuro-protective benefits. ${ }^{29}$ Lithium concentrations above 0.8 $\mathrm{mmol} / \mathrm{l}$ are associated with an increased risk of hypothyroidism and renal impairment especially in women and should be monitored closely. In addition to lithium, there is good evidence for olanzapine, quetiapine, risperidone long-acting injection (LAI) and valproate monotherapy preventing manic relapse, and lamotrigine, quetiapine and lurasidone preventing depressive relapse. ${ }^{17,18,29}$ The SGAs or dopamine antagonists/partial agonists are associated with metabolic side-effects while lamotrigine is associated with serious skin rashes, and dose titration is indicated when initiating therapy with this AED.

\section{Manic episode}

Pharmacological strategies include tapering and discontinuing conventional antidepressants, corticosteroids and stimulants that increase the levels of serotonin, noradrenaline and/or dopamine.

For patients who suffer a manic episode while taking long-term treatment, it is prudent to ensure drug adherence and that the highest recommended dose of SGA or valproate is taken. For patients taking lithium, if plasma levels are at the upper end of the target range $(0.6-0.8 \mathrm{mmol} / \mathrm{l})$, consider adding an SGA or valproate, ${ }^{17}$ because additive efficacy may be achieved through combination therapy targeting both pre- and postsynaptic sites. ${ }^{3,30}$

For patients not already taking long-term treatment, oral administration of a dopamine antagonist achieves a rapid antimanic effect. Trial data suggest that haloperidol, olanzapine, risperidone and quetiapine are particularly effective in short-term reduction of symptoms, while aripiprazole and other dopamine antagonists/partial agonists (paliperidone, ziprasidone, asenapine) are also valuable options. ${ }^{17}$ Valproate is an alternative but should not be used for women of childbearing potential because of its unacceptable teratogenic risks. It should also be noted that valproate is an enzyme inhibitor and the risks for drug-drug interactions are therefore elevated. In particular, it inhibits glucuronidation, which may increase lamotrigine levels significantly. Lithium and carbamazepine may also be considered in this setting.

\section{Depressive episode}

There is insufficient evidence for conventional antidepressants in bipolar depression, possibly indicating an aetiology that is sufficiently distinct from major depressive disorder. ${ }^{31}$ These agents, particularly the dual acting (venlafaxine, duloxetine, amitriptyline and imipramine) may also trigger mania, ${ }^{32}$ and should probably not be prescribed in the absence of a longterm anti-manic agent (lithium, valproate, dopamine receptor antagonist/partial agonist). Only fluoxetine, in combination with olanzapine, has good supporting evidence for efficacy in bipolar depression.

Rather, based on current evidence, quetiapine, olanzapine, lurasidone, or the AED, lamotrigine, are recommended for patients suffering a relapse whilst on long-term treatment, as well as for those not yet taking long-term treatment. ${ }^{17}$ Lithium is considered when symptoms have improved, especially as a prelude to long-term treatment. Continuation treatment of a depressive episode (three months in remission) is usually shorter than for unipolar depression (six months in remission). (Figure 3.)

It should be noted that following discontinuation of antibipolar agents, the risks of relapse and recurrence remain, even after years of sustained remission or recovery. Abrupt lithium withdrawal may lead to early relapse to mania. Discontinuation of any drug should be tapered down over at least 4-8 weeks, 


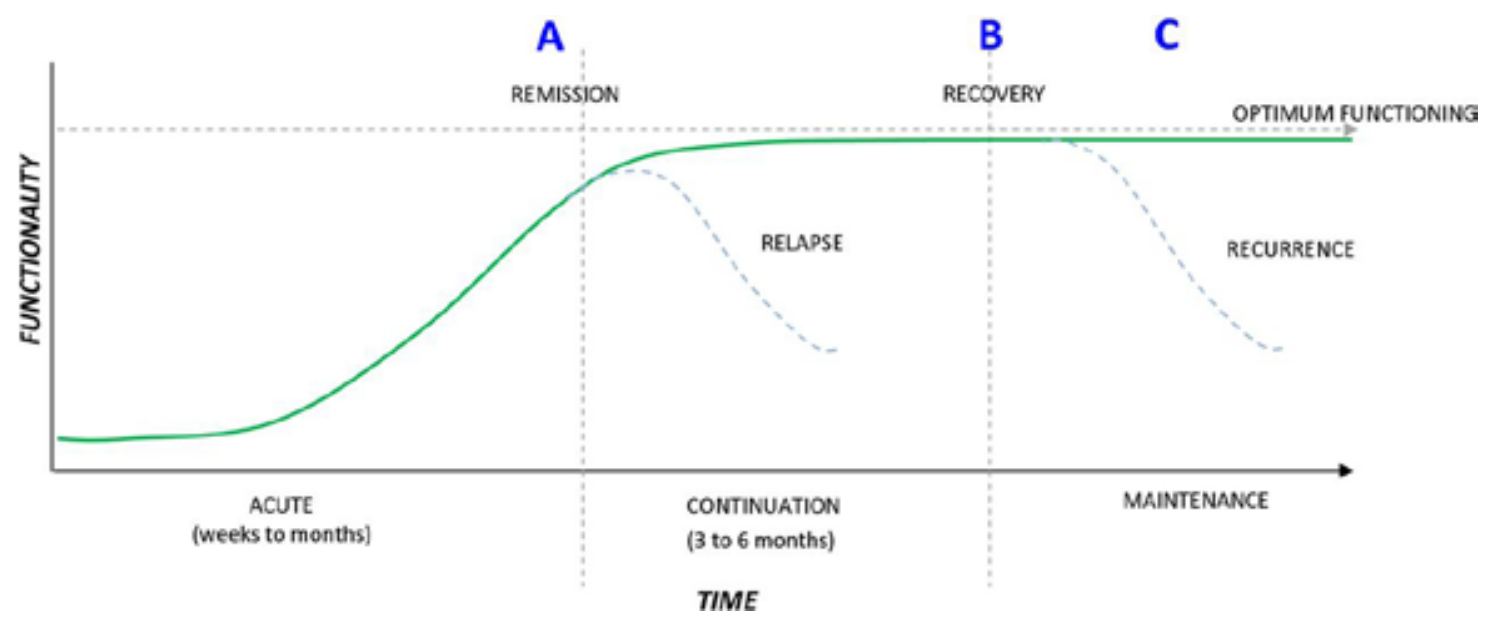

Figure 3. Transition from acute illness through the continuation and maintenance treatment. The acute phase of mania is typically weeks whereas acute depressive episodes may last months. ${ }^{33}$

other (psycho-social) treatment options should remain in place and patients should continue to be monitored regularly, and preferably lifelong. ${ }^{2}$

\section{Conclusion}

Bipolar disorder is a potentially serious illness that may be difficult to manage, yet robust diagnostic criteria and evidence-based treatment recommendations provide a working framework for clinicians and thereby significantly improve outcomes for patients.

\section{References}

1. Ferreira MA, O'Donovan MC, Meng YA, et al. Collaborative genome-wide association analysis supports a role for ANK3 and CACNA1C in bipolar disorder. Nat Genet. 2008;40(9):1056-8.

2. Goodwin GM. Bipolar disorder. Medicine. 2016;44(11):661-3.

3. Goodwin GO. Psychopharmacology CGotBAf. Evidence-based guidelines for treating bipolar disorder: revised second edition-recommendations from the British Association for Psychopharmacology. Journal of Psychopharmacology. 2009;23(4):346-88.

4. Judd LL, Schettler PJ, Solomon DA, et al. Psychosocial disability and work role function compared across the long-term course of bipolar I, bipolar II and unipolar major depressive disorders. J Affect Disord. 2008;108(1):49-58.

5. Marangell LB, Bauer MS, Dennehy EB, et al. Prospective predictors of suicide and suicide attempts in 1,556 patients with bipolar disorders followed for up to 2 years. Bipolar disorders. 2006;8(5p2):566-75.

6. Phillips ML, Kupfer DJ. Bipolar disorder diagnosis: challenges and future directions. The Lancet. 2013;381(9878):1663-71.

7. Association AP. Desk reference to the diagnostic criteria from DSM- $5^{\oplus}$ : American Psychiatric Pub; 2014.

8. Malhi GS, Bassett D, Boyce $P$, et al. Royal Australian and New Zealand College of Psychiatrists clinical practice guidelines for mood disorders. Aust N Z J Psychiatry. 2015;49(12):1087-206.

9. Holmskov J, Licht R, Andersen K, et al. Diagnostic conversion to bipolar disorder in unipolar depressed patients participating in trials on antidepressants. Eur Psychiatry. 2017;40:76-81.

10. Schloesser RJ, Huang J, Klein PS, Manji HK. Cellular plasticity cascades in the pathophysiology and treatment of bipolar disorder. Neuropsychopharmacology. 2008;33(1):110-33.

11. Kim Y, Santos R, Gage FH, Marchetto MC. Molecular Mechanisms of Bipolar Disorder: Progress Made and Future Challenges. Front Cell Neurosci. 2017;11:30.

12. Rainforth $M$, Laurenson M. A literature review of case formulation to inform mental health practice. J Psychiatr Ment Health Nurs. 2014;21(3):206-13.

13. Bender RE, Alloy LB. Life stress and kindling in bipolar disorder: review of the evidence and integration with emerging biopsychosocial theories. Clin Psychol Rev. 2011;31(3):383-98.

14. Oldehinkel AJ, Ormel J, Verhulst FC, Nederhof E. Childhood adversities and adolescent depression: A matter of both risk and resilience. Dev Psychopathol. 2014;26(4pt1):1067-75.

15. Murray $G$, Leitan N, Berk $M$, et al. Online mindfulness-based intervention for late-stage bipolar disorder: pilot evidence for feasibility and effectiveness. J Affect Disord. 2015;178:46-51.
16. Husain MI, Chaudhry IB, Rahman RR, et al. Pilot study of a culturally adapted psychoeducation (CaPE) intervention for bipolar disorder in Pakistan. International Journal of Bipolar Disorders. 2017;5(1):3.

17. Goodwin G, Haddad P, Ferrier I, et al. Evidence-based guidelines for treating bipolar disorder: Revised third edition recommendations from the British Association for Psychopharmacology. Journal of Psychopharmacology. 2016;30(6):495-553.

18. Yatham LN, Kennedy SH, Parikh SV, et al. Canadian Network for Mood and Anxiety Treatments (CANMAT) and International Society for Bipolar Disorders (ISBD) collaborative update of CANMAT guidelines for the management of patients with bipolar disorder: update 2013. Bipolar disorders. 2013;15(1):1-44.

19. Emsley R, Hawkridge S, Potocnik F, et al. Introduction: The South African Society of Psychiatrists (SASOP) Treatment Guidelines for Psychiatric Disorders. South African Journal of Psychiatry. 2013;19(3):134-5.

20. Kendall T, Morriss R, Mayo-Wilson E, Marcus E. Assessment and management of bipolar disorder: summary of updated NICE guidance. BMJ: British Medical Journal. 2014;349.

21. Goldsmith DR, Wagstaff AJ, Ibbotson T, Perry CM. Spotlight on lamotrigine in bipolar disorder. CNS drugs. 2004;18(1):63-7.

22. Li X, Ketter TA, Frye MA. Synaptic, intracellular, and neuroprotective mechanisms of anticonvulsants: are they relevant for the treatment and course of bipolar disorders? Journal of affective disorders. 2002;69(1):1-14.

23. Mathew SJ, Manji HK, Charney DS. Novel drugs and therapeutic targets for severe mood disorders. Neuropsychopharmacology. 2008;33(9):2080-92.

24. Chen G, Zeng WZ, Yuan PX, et al. The Mood-Stabilizing Agents Lithium and Valproate Robustly Increase the Levels of the Neuroprotective Protein bcl-2 in the CNS. Journal of Neurochemistry. 1999;72(2):879-82.

25. Rosenberg G. The mechanisms of action of valproate in neuropsychiatric disorders: can we see the forest for the trees? Cell Mol Life Sci. 2007;64(16):2090-103.

26. Yatham LN, Goldstein JM, Vieta E, et al. Atypical antipsychotics in bipolar depression: potential mechanisms of action. Journal of Clinical Psychiatry. 2005;66(Suppl 5):40-8.

27. Phiel CJ, Zhang F, Huang EY, et al. Histone deacetylase is a direct target of valproic acid, a potent anticonvulsant, mood stabilizer, and teratogen. Journal of Biological Chemistry. 2001;276(39):36734-41.

28. Levin JB, Krivenko A, Howland M, Schlachet $R$, Sajatovic M. Medication Adherence in Patients with Bipolar Disorder: A Comprehensive Review. CNS drugs. 2016;30(9):819-35.

29. Malhi GS, Gessler D, Fritz K, et al. Recommendations in International Clinical Practice Guidelines for Lithium Therapy of Bipolar Disorder. The Science and Practice of Lithium Therapy: Springer; 2017. p. 189-209.

30. Emsley R, Colin F, Flisher AJ, et al. The South African Society of Psychiatrists (SASOP) treatment guidelines for psychiatric disorders. South African Journal of Psychiatry. 2013;19(3):128-99.

31. Liu Y, Blackwood DH, Caesar S, et al. Meta-analysis of genome-wide association data of bipolar disorder and major depressive disorder. Mol Psychiatry. 2011;16(1):2-4.

32. Connolly KR, Thase ME. The clinical management of bipolar disorder: a review of evidence-based guidelines. The primary care companion to CNS disorders. 2011;13(4).

33. Malhi GS, McAulay C, Das P, Fritz K. Maintaining mood stability in bipolar disorder: a clinical perspective on pharmacotherapy. Evidence Based Mental Health. 2014:ebmental-2014-101948. 